\title{
Weitere Untersuchungen über die Wirkung der Ermüdung auf die Reflexe. $\left.{ }^{1}\right)$
}

\author{
Von \\ Dr. Milt. Oeconomakis, \\ Privatdozent an der Universität Athen.
}

(Eingegangen am 9. Juni 1911.)

Durch die Untersuchungen von A uerbach, Knapp und Thomas, Schilling und von mir an Radrennfahrern, Läufern und Wettgehern wurde nachgewiesen, daß hochgradige Ermüdung objektiv nachweisbare Veränderungen der Sehnenreflexe, sowie Ausfallsymptome an den besonders durch die übermäßige Anstrengung betroffenen Körperteilen hervorrufen kann. Bei allen diesen Untersuchungen wurden die Reflexe der oberen Extremitäten nicht berücksichtigt.

Im Folgenden soll nun, durch Untersuchung einer größeren Zahl von Reflexen auch an den oberen Extremitäten, gezeigt werden, wie weit sich die Ausdehnung dieser Veränderungen erstreckt, was einen wesentlichen Beitrag zur Klärung der Frage bieten würde, ob diese Veränderungen einer allgemeinen ätiologischen Einwirkung, etwa einer akut entstehenden Vergiftung durch Ermüdungsstoffe, zuzuschreiben seien, oder ob sie tatsächlich nur den Ausdruck eines lokalen Aufbrauches im Sinne der Edingerschen Theorie darstellen.

Die Gelegenheit zur Ausführung dieser Untersuchungen gab mir wiederum der Marathonlauf bei den im Frühling dieses Jahres (16. April, alten Stils) abgehaltenen Panhellenischen Spielen. Der Lauf geschah wie damals (1906), nur war der Weg heuer wesentlich besser, da er erst im Vorjahr neu chaussiert war.

Der Lauf begann um $3 \mathrm{Uhr}$ nachmittags. Einige Stunden vorher konnten 19 von den 22 Teilnehmern in Marathon untersucht werden. Es wurden bei dieser Untersuchung folgende Reflexe berücksichtigt: Lichtreaktion der Pupillen, Unterkiefer- (Masseter-) Reflex, Triceps-, Biceps-, Beugesehnen- und Radiusreflex an den oberen Extremitäten, Patellar- und Achillessehnenreflex, Oppenheims normaler Unterschenkelreflex, Mendel-Bechterewscher Fußrückenreflex und von den Hautreflexen: Bauchdecken- (mit gesonderter Berücksichtigung

1) Nach einem Vortrag, gehalten in der Athener Ärtztegesellschaft am 4./17. Mai 1911 . 
des epi-, meso- und hypogastrischen Reflexes), Cremaster- und Fußsohlenreflex. Außerdem noch: Pupillenweite oder -differenz, Nystagmus, Rombergsches Zeichen und subjektive Sensibilitätsstörungen.

Von den Ergebnissen dieser Voruntersuchung sind nun folgende hervorzuheben:

Bei zwei dieser Läufer fand sich ein leichtes Schwanken beim Liderschluß (Romberg), und zwei andere boten leichte konjugierte, nystagmusartige Zuckungen beim Blick nach außen. Bei allen war die Lichtreaktion der Pupillen, der Patellar- und Tricepssehnenreflex sowie der epigastrische Bauchreflex ausnahmsweise konstant vorhanden. Auch die Pupillen boten keine sonstige Veränderung. Der Achillessehnenreflex fehlte einseitig bei einem, der sich einige Tage vorher auf der gleichliegenden Seite eine Fußverletzung zugezogen hatte. Ebenso fehlte der Fußsohlenreflex bei einem andern. Bei einem dritten fehlte der Bicepssehnenreflex, der sonst mit auffälliger Regelmäßigkeit bei allen übrigen nachweisbar war.

Von den weniger konstanten und wandelbaren Reflexen fehlten bei diesen 19 Läufen: Der Unterkieferreflex bei 11, der Radiusreflex bei 3, der Beugesehnenreflex oberhalb des Handgelenkes bei 4; diese beiden Reflexe waren auch sonst meistens schwach und bei einem Läufer waren sie nur einseitig vorhanden. Schließlich entbehrten 12 jeder Reflexbewegung bei Prüfung des Unterschenkelreflexes von $O p$ penheim. Der normale Mendel-Bechterewsche Reflex war nur bei 3 Läufern nachweisbar.

Besondere Erwähnung verdient das Verhalten des Cremasterreflexes. Von den 19 untersuchten Läufern entzogen sich der Untersuchung bezüglich dieses Reflexes drei, bei den übrigen 16 wurde wie folgt beobachtet: Der Reflex war normal oder lebhaft nur bei 6 , bei anderen 6 war er schwach, bei einigen sehr schwach, bei einem war er einseitig schwach, bei einem anderen fehlte er einseitig (links), bei zwei weiteren zeigte er sich gar nicht, was bei dem einen (Läufer Nr. 106) sich darauf zurückführen ließ, da $\beta$ der Cremaster sich bei ihm augenscheinlich in einem Kontraktionszustand befand, durch den die Hoden emporgezogen waren. Von den drei, die sich dieser Untersuchung entzogen, konnte der eine, der als Zweiter am Ziel eintraf, mehrere Tage nach dem Lauf untersucht werden, und zeigte sich bei ihm der Reflex einseitig sehr schwach.

Zur Auslösung dieses Reflexes wurden jedesmal alle üblichen Methoden angewandt, d. i. durch Streichen mit dem Stiel des Perkussionshammers in den verschiedenen Höhen der Innenfläche des Oberschenkels (da bemerkt wurde, daß bei einigen das Streichen nicht zu hoch, etwa in der Adductorengegend, sondern viel niedriger erfolgen mußte), sowie durch Kneifen oder Druck über den Condylus internus. Die Hoden 
hingen meistens schlaff herab, und ein Anspannungszustand des Cremasters, wie bei Läufer Nr. 106, durch den eine Arreflexie vorgetäuscht werden konnte, kann bei allen übrigen wohl ausgeschlossen werden. Die Prüfung geschah bei allen in liegender und aufrechter Stellung. Es wurden also alle möglichen Vorsichtsmaßregeln getroffen.

Worauf ist nun die bei den meisten Läufern beobachtete Verminderung oder das Fehlen dieses, sonst bei jungen kräftigen Leuten, im Alter von 19-31 Jahren, fast konstanten und lebhaften Reflexes zurückzuführen? Bekanntlich kann dieser Reflex nach den Angaben von Schönborn bei Gesunden nur in $1 \%$ fehlen, und Steiner hält ihn für mindestens in $97 \%$ konstant. Lokale Affektionen, wie Bruch, Orchitis, Varicocele usw. waren nicht vorhanden. Dieselben sind ja auch derartiger Natur, daß die damit Behafteten auch nicht einmal an den Versuch einer Teilnahme an diesem Lauf denken dürfen.

So kann man also wohl annehmen, daß das Vorhandensein dieser Veränderungen des Reflexes etwas mehr als bloßer Zufall ist, und daß zwischen denselben und dem Lauf irgendwie ein ursächlicher $\mathrm{Zu}$ sammenhang angenommen werden kann. Diese Annahme wird noch besonders durch den Umstand verstärkt, daß mehrere dieser Läufer zwei Tage vorher den $5 \mathrm{~km}$-Lauf mitmachten, außerdem auch die Trainierung zum Lauf durchmachten, und besonders durch die nach diesem Lauf nachgewiesenen Veränderungen an diesem Reflex, die weiter unten zur Erwähnung kommen werden. Besonders auffallend war die Schwäche des Cremasterreflexes vor oder nach dem Laufen gerade bei denjenigen Lüufern, die eine allgemeine Erhöhung aller Reflexe zeigten, denn es trat hier der Gegensatz markanter zutage.

Von den 19 Läufern erreichten 7 das Ziel, das Stadion in Athen. Alle gehörten glücklicherweise zu den vorher in Marathon untersuchten. Von diesen 7 Läufern wurden 6 sofort nach der Ankunft in den Ankleideräumen des Stadions untersucht. Der siebente konnte nach einigen Tagen auch noch untersucht werden. Außerdem kamen noch 3 Läufer, die auf dem Wege zurückblieben und zu Wagen in die Ankleideräume gebracht wurden, zur Untersuchung. Es wurden also im ganzen 9 Läufer gleich nach Beendigung der Leistung untersucht und 3 von ihnen konnten nach einigen Tagen nochmals untersucht werden. Die Untersuchung der letzteren ist also als die genaueste zu bezeichnen und sollen diese an erster Stelle angeführt werden.

Nr. $127^{1}$ ) aus dem Dorfe Chalandri, 22 Jahre alt, Infanterist. 1909 kam er im Marathonlauf als Erster an und nahm auch 1910 wieder teil. Er lief auch den $5 \mathrm{~km}-$ Lauf mit.

Die Untersuchung vor dem Laufe ergab: Kein Romberg. Horizontale nystagmusartige Bewegungen beiderseits beim Blick nach außen. Klagt über

1) Es sind die Nummern, welche die Iäufer bei dem Iaufe trugen. 
Reißen und Brennen an der inneren Fläche der Augenlider. Leichte Conjunctivitis. Pupillen und deren Lichtreaktion normal. Unterkieferreflex nicht vorhanden. Triceps- und Bicepsreflexe normal, Beugesehnenreflex schwach. Radiusreflex fehlt beiderseits. Epi- und mesogastrischer Reflex der Bauchdecken normal, hypogastrischer schwach. Cremaster- und Plantarreflex normal, ebenso Patellarsehnen- und Achillesreflex normal beiderseits. Normaler Unterschenkelreflex von Oppenheim schwach, Fußrückenreflex von Mendel-Bechterew nicht vorhanden.

Als Sieger legte er die ca. $42 \mathrm{~km}$ in 3 Stunden 7 Minuten und 16 Sekunden zurück. Gleich nach seiner Leistung: Gibt an, er habe nur das ,nach solchen Leistungen gewöhnliche Taubheitsgefühl an den Beinen". Kein Romberg. Ny. stagmusartige Bewegungen sind nicht mehr zu konstatieren; es gehe jetzt besser mit den Augen, wie er angibt. Triceps- und Bicepssehnenreflexe normal, Beugesehnen- schwach und Radiusreflex fehlt. Bauchreflex normal, hypogastrischer schwach. Cremasterreflex links vermindert, rechts nicht vorhanden. Patellarreflex beiderseits sehr schwach, mit Jendrassik etwas ausgiebiger, Achillesreflexe gesteigert. Unterschenkelreflex fehlt beiderseits, ebenso der Fußrückenreflex. Plantarreflex rechts gesteigert; beim Streichen der Fußsohle wird die ganze Extremität flektiert und zurückgezogen.

Nach 7 Tagen, am 23. April, stellte er sich wieder zur Untersuchung. Diese ergab: Nystagmusartige Bewegungen wie bei der Untersuchung in Marathon. Patellar- und Achillesreflex beiderseits normal. Unterschenkelreflex schwach vorhanden. Plantarreflex normal, rechts noch immer eine Hyperästhesie der Plantartarhaut wahrnehmbar. Alles übrige wie bei der ersten Untersuchung.

Nr. 110. Kellner in einer Weinstube in Piraeus, 20 Jahre alt, aus Lakonien. Beteiligte sich auch an dem $5 \mathrm{~km}$-Lauf. Vor Beginn: Kein Romberg, kein Nystagmus. Pupillen normal, Lichtreaktion lebhaft. Masseterreflex vorhanden. Reflexe der oberen Extremitäten lebhaft. Alle Bauchdeckenreflexe beiderseits lebhaft. Cremasterreflex schwach. Patellar- und Achillessehnenreflex lebhaft. Unterschenkelreflex von Oppenheim beiderseits vorhanden. Fußrückenreflex schwach. Plantarreflex normal.

Er machte den Lauf in 3 Stunden 12 Minuten, 28 Sekunden und erreichte als Dritter das Stadion. Gleich nach dem Laufe ergab die Untersuchung: Kein Romberg noch Nystagmus. Pupillen normal, Lichtreaktion lebhaft. Masseterreflex normal. Triceps-, Biceps-, Beugesehnen- und Radiusreflex beiderseits lebhaft. Bauchreflexe lebhaft. Cremasterreflex fehlt auf beiden Seiten. Patellarreflex rechts lebhaft, links hochgradig gesteigert; ebenso der Achillesreflex ist links gesteigert: durch leichten Schlag auf alle Höhen der Sehne treten klonusartige Zuckungen ein. Unterschenkelreflex (Oppenheim) links sehr lebhaft, gesteigert; im Gegensatz zu rechts genügt hier ein leichter Strich auf die Innenfläche des Unterschenkels, die Plantarflexion der Zehen lebhaft hervorzurufen. Fußrückenreflex beiderseits schwach. Plantarreflex normal.

Sechs Tage nach dem Lauf wurde er in Piraeus wieder untersucht. Befund: Patellarreflex noch immer bedeutend stärker links, ebenso der Achillesreflex. Unterschenkelreflex links durch viel leichteren Druck stärker als auf der rechten Seite auslösbar. Cremasterreflexe beim Streichen in der Adductorengegend äußerst schwach, fast gleich Null; bei Reizung tiefer gelegener Flächen fast normal. Reflexe der oberen Extremitäten und alles übrige wie bei der ersten Untersuchung. Er klagte über Unwohlsein und Appetitlosigkeit seit dem Tage des Marathonlaufes. Mißbrauch geistiger Getränke negiert. Infolge seines Berufes kommt er fast den ganzen Tag nicht zum Sitzen.

Zwölf Tage nach dem Lauf wurde er in Piraeus nochmals untersucht. Er 
fühlte sich jetzt ganz wohl und erklärte seinen Entschluß, die Trainierung wieder aufzunehmen. Patellar- und Achillesreflex beiderseits gleich lebhaft. Geblieben is t $\mathrm{n}$ ur noch der merkliche Unterschied zwischen den beiderseitigen Unterschenkelreflexen $O$ p penheims. Cremasterreflex normal, scheinbar noch lebhafter als bei der Voruntersuchung in Marathon. An dem übrigen Status keine Veränderung.

Nr. 59. Zeitungsjunge, 21 Jahre alt, aus Gortynia. Er nahm zum erstenmal an einem Wettlauf teil. Voruntersuchung in Marathon: Kein Romberg, kein Nystagmus. Pupilien und deren Lichtreaktion normal. Unterkieferreflex nicht vorhanden. Tricepsreflex beiderseits schwach, Bicepsreflex lebhafter. Beugesehnenund Radiusreflex fehlt auf beiden Seiten. Der Bauchreflex ist nur vom Epigastrium auszulösen. Cremasterreflexe normal. Patellarreflex beiderseits schwach, mit Jendrássik lebhaft. Achillesreflexe schwach. Unterschenkelreflex beiderseits vorhanden. Fußrückenreflex nicht vorhanden. Plantarreflexe schwach.

Er konnte nur $33 \mathrm{~km}$ zurücklegen und gelangte zu Wagen nach dem Stadion. Die Untersuchung in den Ankleideräumen daselbst ergab: Kein Romberg noch Nystagmus. Pupillen und Lichtreaktion derselben normal. Unterkieferreflex fehlt. Tricepssehnenreflexe schwach, Bicepsreflex gesteigert. Beugesehnenund Radiusreflexe fehlen beiderseits. Von den Bauchdeckenreflexen nur der epigastrische auslösbar. Cremasterreflexe normal. Patellarreflexe sind nicht auszulösen, fehlen beiderseits vollständig. Achillesreflex links normal, rechts äußerst schwach. Unterschenkelreflexe normal vorhanden. Mendel-Bechterew fehlt. Plantarreflexe normal.

Eine weitere Untersuchung nach 4 Tagen ergab: Patellarreflexe mit Jendrassik normal. Achillesreflexe gleich schwach. Cremasterreflexe normal. Tricepssehnenreflexe schwach, Bicepsreflex noch immer lebhaft. Bezüglich der übrigen Reflexe genau dasselbe Verhalten wie bei den früheren Untersuchungen.

Hier ist eine Bemerkung über den Gang der Untersuchung einzuschieben. Der Befund bei jeder Untersuchung wurde für alle Läufer besonders in allen Einzelheiten notiert, ohne irgendwelche Berücksichtigung der Resultate der früheren Untersuchungen. Erst nach Beendigung aller Untersuchungen fand das Vergleichen der gemachten Beobachtungen statt. Darauf wurde gehalten, um soviel wie möglich jeder autosuggestiven Einwirkung vorzubeugen. Bei dem Vergleichen der Resultate war nun auffallend, wie sehr sich die Bemerkungen bezüglich der unverändert gebliebenen Reflexe bei allen Untersuchungen desselben Läufers glichen.

Ferner muß bemerkt werden, daß in den Fällen, in denen nach dem Laufe eine Steigerung der Achillesreflexe ohne Klonus verzeichnet wurde, die Sache so liegt, daß der Reflex durch leichtes Schlagen an allen Punkten der Achillessehne und sogar an der Ferse selbst auszulösen war, während das vor dem Lauf nur an einer Stelle der Sehne, gewöhnlich gleich über der Ferse geschah.

Schließlich ist bezüglich des Patellarreflexes noch zu erwähnen, daß neben dem Jendrássikschen Kunstgriff die von Schönborn empfohlene Modifikation desselben, die darin besteht, daß der Läufer die linke Hand des Untersuchers während der Prüfung drücken mußte, 
dabei gute Dienste leistete. Durch dieselbe wurde erreicht, die Reflexe auch dann noch hervorzurufen, wenn alle anderen Methoden versagten.

Nr. 163. 21 Jahre alter Soldat aus Marussi. Vor dem Rennen: Kein Romberg noch Nystagmus. Pupillen und deren Lichtreaktion normal. Unterkieferreflex nicht vorhanden. Triceps- und Bicepssehnenreflex normal, Radius- und Beugesehnenreflexe schwach. Patellar- und Achillesreflex beiderseits normal. Unterschenkel- und Fußrückenreflexe nicht vorhanden. Plantarreflexe schwach. Der Cremaster- sowie die Bauchreflexe wurden nicht untersucht.

Er langte als Zweiter am Ziel an. In den Ankleideräumen blieb er unbeachtet, kam aber nach 10 Tagen zur Untersuchung. Er zeigte folgendes: Hinken. Nach dem $33 . \mathrm{km}$ wurde er, wie er sagt, von Schmerz am linken Bein befallen und kam infolgedessen hinkend ins Stadion. Schmerzhafte Schwellung an der Außenfläche des unteren Drittels des linken Beins bis zur oberen Seite des Fußes; die Gegend der Achillessehne ist ebenfalls geschwollen und schmerzhaft bei Druck. Achillesreflex links äußerst $\mathrm{schwach}$, rechts normal. Plantarreflexe links gesteigert; bei der Prüfung kraftvolles Zurückziehen des ganzen Beins. Patellarreflex auf beiden Seiten lebhaft. Bauchreflexe nicht auszulösen (Muskelspannung!). Cremasterreflex links sehr schwach, rechts normal. Alles übrige genau wie bei der Untersuchung in Marathon.

Nr. 168. 19 Jahre alt, Arbeiter aus dem Dorfe Marussi. Nahm 4 Tage vorher am $18 \mathrm{~km}$-Lauf teil. Vor dem Rennen: Leichtes Schwanken beim Augenschluß. Kein Nystagmus. Pupillen und deren Lichtreaktion normal. Unterkieferreflex nicht vorhanden. Triceps-, Biceps-, Radius- und Beugesehnenreflexe schwach. Von den Bauchdeckenreflexen nur der epigastrische normal, die übrigen schwach und leicht erschöpfbar. Cremasterreflexe normal. Patellarreflex normal, Achillesreflexe schwächer. Unterschenkelreflex vorhanden, Fußrückenreflex dagegen nicht. Plantarreflexe gesteigert.

Erreichte als Vierter das Ziel. Nach dem Rennen: Leichter Romberg. Kein Nystagmus. Reflexe der oberen Extremitäten .gleichmäßig schwach. Cremasterreflex beiderseits schwach. Patellarreflex links schwächer als rechts. Achillesreflex rechts gesteigert, links normal. Unterschenkelreflex von oppenheim links nicht vorhanden. Mendel-Bechterew fehlt. Plantarreflex beiderseits schwach. Pupillen, Masseter- und Bauchreflex wie vorher.

Nr. 176. 31 Jahre alt, Ackerbauer aus Almyros. Vor dem Rennen: Leichter Romberg. Kein Nystagmus. Pupillen und Lichtreaktion normal. Unterkieferreflex vorhanden. Triceps- und Bicepsreflex normal. Beugesehnen- und Radiusreflex fehlen beiderseits. Bauchreflexe lebhaft. Cremasterreflex normal. Patellarreflex beiderseits lebhaft. Achillesreflexe normal. Normaler Oppenheim links schwach, rechts fehlt. Fußrückenreflex fehlt. Plantarreflexe schwach.

Er konnte als Fünfter das Stadion erreichen. Gleich darauf: Leichter Rom berg. Kein Nystagmus. Pupillen und Lichtreaktion normal. Masseterreflex normal vorhanden. Triceps-, Biceps-, Beugesehnen- und Radiusreflexe normal. Bauchreflexe alle lebhaft vorhanden. Cremasterreflex beiderseits sehr schwach. Patellarreflexe äuBerst schwach, links nur mit Kunstgriff und dann noch sehr schwach und nicht bei jedem Schlag auslösbar. Oppenheim fehlt beiderseits. Fußrückenreflex nicht vorhanden. Achillessehnenreflex links vermindert. Plantarreflexe schwach.

Nr. 153. 26 Jahre alter Student aus Smyrna. Sieger 2 Tage vorher im $5 \mathrm{~km}$ Lauf. Vor dem Rennen: Kein Romberg, kein Nystagmus. Pupillen normal, Lichtreaktion lebhaft. Unterkieferreflex vorhanden. Reflexe der oberen Extremitäten, besonders Triceps- und Bicepsreflex in normaler Stärke. Bauchreflex 
nur vom Epigastrium. Cremasterreflex rechts normal, links nicht vorhanden. Patellarreflexe normal. Achillesreflexe lebhaft. Unterschenkel- und Fußrückenreflex nicht vorhanden. Plantarreflexe normal.

Nach dem Rennen: Kein Romberg, kein Nystagmus. Pupillen, Licht- und Unterkieferreflex normal. Triceps- und Bicepsreflexe normal. Radius- und Beugesehnenreflexe fehlen. Epigastrischer Bauchreflex normal, übrige nicht vorhanden. Cremasterreflex fehlt beiderseits. Patellarreflexe schwach, Achillesreflexe dagegen lebhaft. Unterschenkelreflex rechts vorhanden, links fehlt, sowie Fußrückenreflex. Plantarreflexe normal.

Nr. 189. 20 Jahre alt, Student. Beteiligte sich auch am $5 \mathrm{~km}-\mathrm{Lauf}$. Vor dem Rennen: Kein Romberg, kein Nystagmus. Pupillen normal, Lichtreaktion lebhaft. Unterkiefereflexe lebhaft. Reflexe der oberen Extremitäten normal vorhanden. Bauchdecken und Cremasterreflex lebhaft. Patellar- und Achillesreflexe gesteigert. Oppenheim vorhanden. Mendel-Bechterew fehlt. Plantarreflexe normal.

Gleich nach dem Lauf: Leichter Romberg. Kein Nystagmus. Pupillen und Lichtreaktion normal. Unterkieferreflex lebhaft. Reflexe der oberen Extremitäten normal vorhanden. Alle Bauchreflexe lebhaft. Cremasterreflex lebhaft. Patellar- und Achillesreflex beiderseits gesteigert. Oppenheim beiderseits vorhanden, Mendel-Bechteren fehlt. Plantarreflex gesteigert; Hyperästhesie der Plantarhaut. Klagen über ziehende Schmerzen an den Beinen und Brennen an den Sohlen.

Nr. 200. 20 Jahre alt, Angestellter der elektrischen Gesellschaft in Patras. Nahm auch am $5 \mathrm{~km}$-Lauf 2 Tage vorher teil.

Vor dem Rennen: Kein Romberg, kein Nystagmus. Pupillen und Lichtreaktion normal. Unterkieferreflex nicht vorhanden. Triceps-, Biceps- und Radiusreflexe normal, Beugesehnenreflexe fehlen. Bauchreflexe alle normal vorhanden. Cremasterreflex beiderseits schwach. Patellar- und Achillesreflex normal. Oppenheim sowie Mendel-Bechterew nicht vorhanden. Plantarreflexe lebhaft.

Er konnte nur $31 \mathrm{~km}$ zurücklegen. Gleich nach der Ankunft im Stadion: Reflexe der oberen Extremitäten alle normal vorhanden. Cremasterreflexe schwach. Patellarreflex rechts stark vermindert, nur mit Jendrássik-Schönborn erzielbar. Achillesreflexe schwach. Plantarreflex normal. Alles übrige genau wie vor dem Lauf.

Nr. 82. Fabrikarbeiter aus Piraeus, 27 Jahre alt. Vor dem Rennen: Kein Romberg, kein Nystagmus. Pupillen und Lichtreaktion normal. Unterkieferreflex nicht vorhanden. Reflexe der oberen Extremitäten gleichmäßig schwach. Von den Bauchdeckenreflexen fehlt nur der hypogastische. Cremasterreflexe normal. Patellar- und Achillesreflexe normal. Unterschenkel- sowie Fußrückenreflex nicht vorhanden. Plantarreflexe schwach.

Nach der Ankunft im Stadion: Reflexe der oberen Extremitäten schwach, mit Ausnahme des Bicepsreflexes, der gesteigert. Bauchreflex nur vom Epigastrium normal auslösbar. Cremasterreflex rechts schwach, links nicht vorhanden. Patellarreflexe rechts stark vermindert, links fast Null: nur mit Kunstgriff und auch dann noch sehr schwach. Achillesreflexe normal. Plantarreflexe schwach. Alles übrige wie bei der Voruntersuchung.

Aus all den obigen Beobachtungen geht nun klar hervor, daß auch dieses Mal nach dem Laufen auffällige Veränderungen der Reflexe festzustellen waren, die der übermäßigen körperlichen Anstrengung infolge des Laufens zuzuschreiben sind. Diese Veränderungen wurden fast ausschließlich an den Reflexen der unteren Extremitäten konstatiert, d. i. an denjenigen Körperteilen, denen die Hauptarbeit zufiel. 
So war der Patellarreflex bei diesen Läufern:

bei einem einseitig gesteigert,

, dreien , vermindert,

, dreien beiderseits sehr schwach,

, einem ,, erloschen

und blieb nur bei einem unverändert.

Der Achillessehnenreflex fand sich

$$
\begin{gathered}
\text { bei einem einseitig gesteigert, } \\
\text {, zweien , vermindert. } \\
\text {, zweien beiderseits gesteigert, } \\
\text {, einem , sehr schwach }
\end{gathered}
$$

und blieb bei dreien unverändert.

Einige bemerkenswerte Änderungen zeigten sich auch bei dem normalen Unterschenkelreflex von Oppenheim, welche, abgesehen von einigen nebensächlichen Differenzen, wie folgt zusammengefaßt werden können: Der Reflex schien den Veränderungen der beiden vorher besprochenen zu folgen, da er sich bei zwei Läufern mit einseitiger Steigerung des Patellar- und Achillesreflexes auf der gleichen Seite gesteigert darstellte, bei zwei Läufern dagegen mit verminderten Patellarreflexen nach dem Laufe vollständig ausblieb.

Auffallend verändert zeigte sich außerdem der Cremasterreflex, d. i. er war

bei zwei vermindert auf beiden Seiten,

„zwei fehlte einseitig,

, zwei fehlte beiderseits,

,, dreien blieb er unverändert.

Der Plantarreflex zeigte eine Steigerung mit lebhafter Hyperästhesie der Plantarhaut bei drei Läufern,Verminderung bei zweien und blieb unverändert bei den übrigen.

Der Fußrückenreflex zeigte keine Veränderung.

Bei den übrigen Reflexen der oberen Extremiäten und des Rumpfes wurde, wenn man von der in zwei Fällen beobachteten Steigerung des Bicepsreflexes und von den in drei weiteren Fällen konstatierten kleinen Veränderungen der Radius- und Beugesehnenreflexe absieht, keine erwähnenswerte Veränderung wahrgenommen. Die Veränderungen der letztgenannten Reflexe bestanden darin, daß3 sie in zwei Fällen erst nach dem Laufen auftraten und in einem anderen dritten nicht mehr auszulösen waren.

Der Tricepssehnenreflex aber, der konstanteste aller Reflexe der oberen Extremitäten, zeigte bei keinem Läufer irgendwelche Veränderung.

Ein solches Fehlen einer ausgesprochenen Veränderung bei den übrigen Reflexen trat noch prägnanter bei dem Läufer $\mathrm{Nr} .59$ hervor, 
bei dem es mit dem höchsten Grad der Veränderung am Patellarreflex, d. h. dessen völligem Ausbleiben, zusammen auftrat.

Ein Schwanken des Körpers beim Liderschluß wurde, wie erwähnt, bei drei Läufern beobachtet, bei einem erst nach dem Laufen, bei zweien auch schon vorher und blieb nach dem Laufe bei.

Die Pupillen und ihre Lichtreaktion zeigten keine Veränderung. Zwar wurden bei einigen Läufern mäßige Pupillenerweiterungen und Verminderung der Lichtreaktion beobachtet, sie wurden jedoch in den Beobachtungen nicht erwähnt, da sie einfach auf die verschiedenen Lichtverhältnisse zurückzuführen sind, denn während die Untersuchung in Marathon bei vollem Tageslicht geschah, mußte die Untersuchung nach dem Laufe wegen der inzwischen eingetretenen Dunkelheit bei künstlichem Licht vorgenommen werden. Bemerkenswert ist noch, daß die bei dem Läufer $\mathrm{Nr}_{\text {; }} 127$ vor dem Lauf beobachteten nystagmusartigen Bewegungen nach dem Laufe fehlten. Eine Erscheinung, die wohl auf einen Zufall zurückzuführen sein möchte. Jedenfalls ließ sich eine genaue Untersuchung des Auges und dessen Hintergrundes nicht durchführen.

Der Unterkieferreflex blieb unverändert. Einige Läufer klagten über Taubheitsgefühl an den Beinen und Brennen an den Fußsohlen. Pathologische Reflexe, wie z. B. Oppenheim und Babinski, wurden nicht beobachtet.

Wie lassen sich nun alle diese Phänomene erklären?

Die Annahme einer Vergiftung durch bei der Ermüdung entstehende Toxine steht mit den Ergebnissen obiger Untersuchung nicht im guten Einklang, denn eine solche Toxämie, als eine allgemeine Schädigung des Organismus, hätte wohl auch auf alle übrigen Reflexe in gleichem Maße einwirken und allgemeinere Erscheinungen zur Folge haben müssen. Die ungleiche Verteilung der Veränderungen, das Unveränderte der übrigen Reflexe, besonders des Tricepssehnenreflexes an den oberen Extremitäten, während die der unteren fast ausschließlich Veränderungen erlitten, erklärt sich dagegen am besten durch die von Edinger vertretene Anschauung des lokalen Nervenaufbrauches.

Die Möglichkeit einer materiellen Schädigung durch Utberfunktion in einem bestimmten Abschnitt des Nervensystems trägt schon seit lange den Stempel des experimentellen Beweises (Edinger, Holmes u. a.). Eine solche Schädigung ist sehr wohl auch in unseren Fällen verständlich und annehmbar, wenn man nur die funktionelle Überspannung in Rechnung zieht, welcher der Reflexbogen des Patellarund Achillesreflexes in erster Linie beim Vorgange des andauernden Laufens unterworfen ist.

Es wäre dann auch die dauernde Anspannung des Cremasters oder vielleicht noch mehr die fortwährende Reibung der Hoden an den Innen- 
flächen der Schenkel während des Laufens als das schädigende Moment für die bei unseren Läufern beobachteten Veränderungen des Cremasterreflexes anzusehen. Für eine solche Schädigung im sensorischen Teil des Reflexbogens spricht übrigens der Umstand, daß bei einigen Läufern der beim Streichen in der Adductorengegend ausbleibende Reflex jedoch durch Reizung tiefer gelegener Partien der Innenfläche des Schenkels auszulösen war.

In ähnlichem Sinne könnte man auch eine Erklärung für die bei zwei Läufern nach dem Laufe beobachtete Steigerung der Bicepssehnenreflexe versuchen. Dabei ist nur zu berücksichtigen, daß die Läufer fast während des ganzen Laufes die oberen Extremitäten in rechtwinkliger Beugung und angezogen, die Fäuste geballt oder darin einen Gegenstand halten, um durch den Druck auf denselben die Spannkraft zu vermehren. Fs wurden ja außerdem auch einige Veränderungen an den Beugesehnenreflexen wahrgenommen, die auch, wenn man sie, trotz der gewöhnlichen Inkonstanz und Wandelbarkeit dieser Reflexe, überhaupt in Betracht ziehen will, für obige Erklärung sprechen.

Die Veränderungen endlich des Fußsohlenreflexes wurden schon von Knapp und Thomas wohl mit Recht auf eine durch das Laufen bedingte mechanische Irritation der Plantarhaut zurückgeführt.

Wenn man somit den ätiologischen Einfluß des örtlichen Nervenaufbrauches anerkennt, kommt man in die Lage, die oben festgestellten Erscheinungen auf die der Wirklichkeit am meisten entsprechende Weise zu erklären.

Damit soll aber die mögliche Hervorrufung einer Toxämie durch die Ermüdung durchaus nicht in Abrede gestellt werden. Eine solche wurde ja auch durch die von Baldes, Heichelheim und Metzger, Arnaud und Bordas ${ }^{1}$ ) an Wettgehern und von Kuzis und Sotiriadis $^{1}$ ) an Läufern vorgenommenen Urinuntersuchungen bewiesen. Aber auf Rechnung der Toxämie können die Reflexveränderungen, wie sie hier konstatiert wurden, nicht gesetzt werden.

Nervenaufbrauch und Toxämie durch übermäßige körperliche Anstrengung können übrigens sehr wohl nebeneinander bestehen. Ja es wird interessant sein, nachzuweisen, inwiefern diese Toxämie ihrerseits als ersatzstörendes Moment, im Sinne der Edingerschen Theorie, einwirken kann, d. i. inwieweit dem Vorhandensein oder dem Fehlen einer solchen endogenen Vergiftung, oder ihren verschiedenen Graden, die bei dem Ausgleich der Aufbrauchserscheinungen während der Erholung beobachteten individuellen Untersehiede zuzuschreiben sind. Wie bekannt, geschieht dieser Ausgleich bei den meisten schon innerhalb des nächsten Tages, während er bei anderen, wie bei unserem Läufer Nr. 110, mehrere Tage auf sich warten läßt.

1) Mündliche Mitteilungen. 
Eine systematische Urinuntersuchung, die parallel mit der wiederholten Prüfung der nervösen Aufbrauchserscheinungen bei jedem Läufer zu geschehen hätte, würde viel zur Aufklärung dieser Frage beitragen.

\section{Literaturverzeichnis.}

A uerbach, Neurologische Untersuchungen an Radrennfahrern. Neurol. Centralbl. 1905, Nr. 6.

Baldes, Heichelheim und Metzger, Untersuchungen über den Einfluß von Körperanstrengungen auf Zirkulationsapparat, Nieren und Nervensystem (Schilling). Münch. med. Wochenschr. 1906, Nr. 38.

Edinger, Der Anteil der Funktion an der Entstehung von Nervenkrankheiten. Wiesbaden 1908.

Knapp und Thomas, The Reflexes in long distance runners. Journ. of nerv. and ment. diseases. 1904. Januar.

Lewandowsky, Handbuch der Neurologie, 1. 1910.

Oppenheim, Lehrbuch der Nervenkrankheiten, 1908.

Oeconomakis, Die Sehnenreflexe angestrengter Körperteile. Untersuchungen an Marathonläufern. Neurol. Centralbl. 1907, Nr. 11 und 12. 